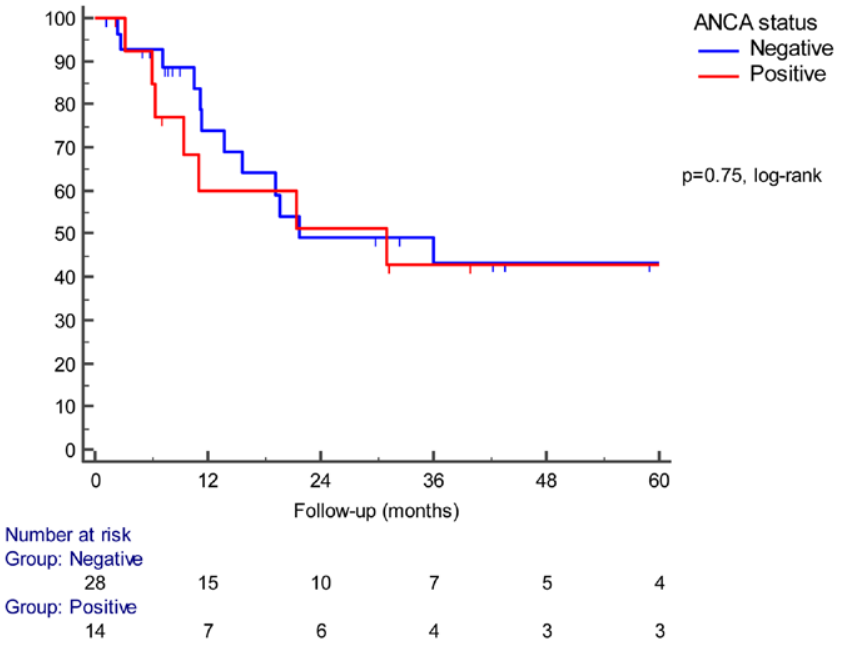

Figure 1. Kaplan-Meier relapse-free survival curve according to the ANCA status

Disclosure of Interests: None declared

DOI: 10.1136/annrheumdis-2021-eular.3261

\section{POS0252 IMPACT OF ANCA-ASSOCIATED VASCULITIS ON CLINICAL OUTCOMES AND RESOURCE UTILIZATION IN ELECTIVE PCI}

K. Mathias ${ }^{1}$, L. Mathias ${ }^{2}$, A. Mantha ${ }^{3}$, D. Arkfeld ${ }^{2} .{ }^{1}$ University of Chicago, Internal Medicine, Chicago, United States of America; ${ }^{2}$ USC Keck School of Medicine, Rheumatology, Los Angeles, United States of America; ${ }^{3}$ USC Keck School of Medicine, Internal Medicine, Los Angeles, United States of America

Background: Anti-neutrophil cytoplasmic antibody (ANCA) associated vasculitides (AV) can increase risk of atherosclerosis. ${ }^{1}$ Patients with autoimmune disease have increased rates of coronary events that are not fully explained by classic risk factors. ${ }^{2} \mathrm{~A}$ prior study has shown that autoimmune vasculitides -including polyarteritis nodosa, hypersensitivity granulomatosis with polyangiitis, giant cell arteritis, thrombotic angiitis, Takayasu arteritis, Goodpasture syndrome, and Kawasaki disease -are associated with increased cost of care and increased 30-day readmission after percutaneous coronary intervention (PCI). ${ }^{3}$ It is important to distinguish the cardiovascular outcomes of small-vessel ANCA-associated vasculitides from the other vasculitides included in this prior study because coronary arteritis is typically a complication of medium to large vessel vasculitis. Objectives: To investigate the impact of comorbid ANCA-associated small vessel vasculitis $(\mathrm{AV})$ on outcomes of $\mathrm{PCl}$ in a nationally representative cohort. Methods: Patients undergoing PCI in the 2016-2018 National Inpatient Sample were selected and those with ANCA-associated vasculitis were identified using the ICD-10 classification system. The NIS is an all-payer, survey-weighted, inpatient database estimating 37 million annual U.S. hospitalizations managed by the Healthcare Cost and Utilization Project (HCUP). Primary study outcomes were prevalence of comorbid vasculitis, mortality, complications, and cost of care.

Results: Of 1,351,670 patients undergoing PCl were identified, $410(0.03 \%)$ had diagnosis of ANCA-vasculitis. AV patients were similar to non-AV patients in distribution of gender, race, and median household income. AV patients were older $(70.9$ vs 65.2 years, $\mathrm{P}<0.001)$ and more likely to utilize Medicare $(78 \%$ vs $53 \%, \mathrm{P}<0.005)$. AV patients were more likely to present with non-ST segment myocardial infarction (NSTEMI) $(57.3 \%$ vs $41.5 \%, P<0.005)$ and less likely to present with ST-elevation myocardial infarction (NSTEMI) $(18.3 \%$ vs $31.3 \%$, $\mathrm{P}<0.05)$. AV patients had similar adjusted mortality $(6.1 \%$ vs $2.6 \%$, aOR: 2.1 , $P=0.8)$, cost of care $(\$ 32,985$ vs $\$ 25,767, \beta=0.02, P=0.7)$, and length of hospitalization (6.0 vs 3.9 , IRR: $1.2, \mathrm{P}=0.13$ ).

Conclusion: The presence of ANCA-associated vasculitis did not appear to negatively impact inpatient clinical outcomes or utilization of care among patients undergoing $\mathrm{PCl}$ in the United States. Cost of care was not increased, in contrast to an increase cost of care when small, medium, and large vasculitides are grouped together. The greater proportion of NSTEMI patients among those with AAV corresponds with a growing body of literature implicating inflammatory processes in the pathogenesis of certain forms of myocardial infarction meriting further investigation.

REFERENCES:

[1] Misra DP, Shenoy SN. Cardiac involvement in primary systemic vasculitis and potential drug therapies to reduce cardiovascular risk. Rheumatol Int. 2017;37(1):151-167. doi:10.1007/s00296-016-3435-1.
[2] Amaya-Amaya J, Sarmiento-Monroy JC, Rojas-Villarraga A. Cardiovascular Involvement in Autoimmune Diseases. El Rosario University Press; 2013. Accessed February 1, 2021. https://www.ncbi.nlm.nih.gov/books/ NBK459468/.

[3] Bailey KL, Mantha A, Sanaiha Y, Mathias L, Benharash P, Ebrahimi R. Short-Term Outcomes After Percutaneous Coronary Intervention in Patients With Autoimmune Vasculitides. J Am Coll Cardiol. 2019;74(1):159-160. doi:10.1016/j.jacc.2019.04.051.

Disclosure of Interests: None declared

DOI: 10.1136/annrheumdis-2021-eular.3369

\begin{tabular}{|l|l|}
\hline POS0253 & RITUXIMAB-BIOSIMILAR FOR ANTI-NEUTROPHIL \\
CYTOPLASMIC ANTIBODIES-ASSOCIATED \\
VASCULITIS: EXPERIENCE OF A SINGLE ITALIAN \\
CENTER
\end{tabular}

S. Sartorelli ${ }^{1}$, A. Cariddi ${ }^{1}$, L. Moroni ${ }^{1,2}$, E. Baldissera ${ }^{1}$, E. Bozzolo ${ }^{1}$, L. Dagna ${ }^{1,2}$. ${ }^{1}$ IRCCS San Raffaele Hospital, Unit of Immunology, Rheumatology, Allergy and Rare Diseases, Milan, Italy; ${ }^{2}$ Vita-Salute San Raffaele University, School of Medicine, Milan, Italy

Background: Rituximab (RTX), an anti-CD20 monoclonal antibody, represents a valuable treatment for anti-neutrophil cytoplasmic antibodies (ANCA)-associated vasculitis (AAV). RTX biosimilar CT-P10 (RTX-B) has been approved in Europe in all indications held by RTX originator (RTX-O). As stated by recent international consensus-based recommendations, ${ }^{1}$ there is evidence regarding safety and efficacy of biosimilars in the context of rheumatic diseases, but it is encouraged to gather additional data. ${ }^{2,3}$

Objectives: To report the experience of a single Italian center with RTX-B in AAV in terms of safety and efficacy.

Methods: We retrospectively reviewed the charts of all AAV patients followed up in our Small Vessel Vasculitis Clinic and we selected those who received RTX-B between October 2017 and May 2020, both naïve to RTX (RTX-Bn) or already treated with $\geq 1$ course of RTX-O and switched to RTX-B (RTX-Bs). Baseline features, disease outcome, concomitant therapy and adverse events 6 (T6), 12 (T12) and 24 (T24) months after RTX-B introduction, when available, were collected. Non-parametric statistic tests were used.

Results: Fifty-six AAV patients (44 [78.6\%] granulomatosis with polyangiitis (GPA), 12 [21.4\%)] microscopic polyangiitis (MPA)) received RTX-B with a median follow up of 20 (IQR 10-24) months. ANCA were positive in 49 (87.5\%) patients. Ten (17.9\%) patients were newly diagnosed with AAV, while $23(41.1 \%)$ had refractory disease and another 23 had relapsing disease.

Thirty-three (58.9\%) patients were RTX-Bs, whereas $23(41.1 \%)$ RTX-Bn. In $29(51.8 \%)$ patients RTX was decided because of remission induction, while in $27(48.2 \%)$ as maintenance regimen. Median cumulative RTX-B dose was 2.5 (2-3.875) grams with 3 (IQR 2-4) median courses. AAV activity, adverse events and concomitant therapy at T6, T12 and T24 are shown in Table 1. One GPA patient died of severe infection 3 months after a single infusion of RTX-B $500 \mathrm{mg}$ as maintenance therapy.

Considering the $52(92.6 \%)$ patients who reached a 6 months-follow up, we marked a significant decrease of number of patients on steroid therapy (39 [75\%] vs 27 [51.9\%]; $p=0.003$ ), of median prednisone daily dose (5 [IQR 5-25] vs 2.5 [IQR $1.25-5 \mathrm{mg} ; \mathrm{p}=0.001$ ) and of number of patient on concomitant bDMARDs (10 [19.2\%] vs $5[9.6 \%] ; p=0.003)$.

Analysing the $40(71.4 \%)$ patients who reached a 12 months-follow up, we confirmed the tendency with a decrease of number of patients on steroid therapy (29 [72.5\%] vs 13 [32.5\%]; $p=0.007)$, of median prednisone daily dose (5 [IQR $5-12.5]$ vs 3.75 [IQR 2.75-7.5]; $p=0.008$ ) and of number of patient on concomitant bDMARDs (9 [22.5\%] vs $3[7.5 \%] ; p=0.009)$.

No statistically significant difference was found for the same variables comparing T0 versus T24.

Table 1. AAV activity, concomitant therapy and adverse events at T0, T6, T12 and T24.

\begin{tabular}{lcccc}
\hline & T0 & T6 & T12 & T24 \\
\hline $\mathrm{n}(\%)$ & $56(100)$ & $52(92.6)$ & $40(71.4)$ & $18(32.1)$ \\
Dose RTX-B, median (IQR) grams & $1.75(0.5-2)$ & $0.5(0.5-1)$ & $0.5(0.5-1)$ & $0.5(0.5-1)$ \\
Concomitant prednisone therapy, n (\%) & $42(75)$ & $27(48.2)$ & $13(32.5)$ & $4(22.2)$ \\
Dose prednisone, median (IQR) & $5(5-15.625)$ & $2.5(2.5-5)$ & 3.75 & 2.75 \\
$\quad$ milligrams/day & & & $(2.75-7.5)$ & $(1.56-4.5)$ \\
Concomitant bDMARDs, n (\%) & $10(17.9)$ & $5(9.6)$ & $3(7.5)$ & $1(5.6)$ \\
Refractoriness & $\mathrm{NA}$ & $4(7.7)$ & $2(5)$ & $1(5.6)$ \\
Relapse & $\mathrm{NA}$ & $0(0)$ & $3(7.5)$ & $2(11.1)$ \\
Remission & $\mathrm{NA}$ & $48(92.3)$ & $35(87.5)$ & $15(83.3)$ \\
Suspension & $\mathrm{NA}$ & $10(19.2)$ & $9(22.5)$ & $4(22.2)$ \\
Infusion reaction, n (\%) & $1(1.8)$ & $1(1.9)$ & $0(0)$ & $0(0)$ \\
Infectious adverse events, n (\%) & $\mathrm{NA}$ & $10(19.2)$ & $6(15)$ & $3(16.7)$ \\
Severe infectious adverse events, n (\%) & $\mathrm{NA}$ & $2(3.8)$ & $1(2.5)$ & $0(0)$ \\
\hline
\end{tabular}


Conclusion: Our experience with RTX-B is limited; however, it represents an extensive report about the use of RTX-B in AAV patients and confirmed that it may be a well-tolerated and effective therapy in AAV setting, in agreement with previous evidence on RTX-O. Prospective and larger studies are needed to confirm this preliminary evidence.

REFERENCES:

[1] Kay J, et al. Ann Rheum Dis 2018;77:165-74.

[2] Kwon HC, et al. Yonsei Med J 2020;61:712-9.

[3] Mittal S, et al. Clin Rheumatol 2021;40:645-51.

Disclosure of Interests: Silvia Sartorelli: None declared, adriana cariddi: None declared, Luca Moroni: None declared, Elena Baldissera Speakers bureau: Pfizer, Roche, Sanofi-Genzyme, Consultant of: Novartis, Enrica Bozzolo: None declared, Lorenzo Dagna Consultant of: Abbvie, Amgen, Biogen, BristolMyers Squibb, Celltrion, Galapagos, GlaxoSmithKline, Novartis, Pfizer, Roche, Sanofi-Genzyme, and SOBI., Grant/research support from: The Unit of Immunology, Rheumatology, Allergy and Rare Diseases (UnIRAR) received unrestricted research/educational grants from Abbvie, Bristol-Myers Squibb, Celgene, GlaxoSmithKline, Janssen, Merk Sharp \& Dohme, Mundipharma Pharmaceuticals, Novartis, Pfizer, Roche, Sanofi-Genzyme, and SOBI.

DOI: 10.1136/annrheumdis-2021-eular.1672

\section{POS0254 EFFICACY OF APREMILAST IN THE TREATMENT OF ORAL ULCERS OF BEHÇET'S SYNDROME: RESULTS FROM THE EUROPEAN SUBGROUP OF RELIEF}

A. Mahr ${ }^{1}$, G. Hatemi ${ }^{2}$, M. Takeno ${ }^{3}$, D. $\mathrm{Kim}^{4}$, M. Melikoglu ${ }^{1}$, D. Saadoun ${ }^{5}$, C. C. Zouboulis ${ }^{6}$, S. Cheng ${ }^{7}$, S. Richter ${ }^{8}$, S. Jardon ${ }^{8}$, M. Paris ${ }^{8}$, M. Chen ${ }^{8}$, Y. Yazici ${ }^{9} .{ }^{1}$ Cantonal Hospital St. Gallen, Department of Rheumatology, St. Gallen, Switzerland; ${ }^{2}$ sstanbul University - Cerrahpasa, Department of Internal Medicine, Istanbul, Turkey; ${ }^{3}$ Nippon Medical School, Musashi Kosugi Hospital, Department of Allergy and Rheumatology, Kawasaki, Japan; ${ }^{4}$ Yonsei University College of Medicine and Severance Hospital, Associate Professor, Hepotolgy, Seoul, Korea, Rep. of (South Korea); ${ }^{5}$ Pitié-Salpêtrière Hospital, Department of Internal Medicine and Clinical Immunology, Paris, France; ${ }^{6} 6$ Dessau Medical Center, Brandenburg Medical School Theodor Fontane, Director of the Departments of Dermatology, Venereology, Allergology and Immunology, Dessau, Germany; ${ }^{7}$ Amgen, Inc., Global Medical Affairs, Director Biostats, Thousand Oaks, CA, United States of America; ${ }^{8}$ Amgen, Inc., Global Medical Affairs, Thousand Oaks, CA, United States of America; ${ }^{9}$ New York University School of Medicine, Department of Rheumatology, New York, NY, United States of America

Background: Behçet's syndrome, a chronic, multi-system variable vessel vasculitis, is often characterized by painful oral ulcers (OU) affecting quality of life (QoL). Apremilast (APR), an oral PDE4 inhibitor, demonstrated efficacy in OU treatment in the phase 3 multinational RELIEF study.

Objectives: To evaluate APR efficacy in OU treatment in patients with active Behçet's syndrome in a prespecified subgroup of patients enrolled in 13 European RELIEF sites (France, Germany, Greece, and Italy).

Methods: patients were adults with active Behçet's syndrome and $\geq 3$ OU at randomization or $\geq 2 \mathrm{OU}$ at screening and randomization, without active major organ involvement. Patients were randomized (1:1) to APR $30 \mathrm{mg}$ BID or PBO during a 12-week double-blind phase. The primary endpoint was area under the curve for the number of $\mathrm{OU}$ through Week $12\left(\mathrm{AUC}_{\mathrm{Wk} 0-12}\right)$. Other outcomes were OU pain visual analog scale (VAS); achievement of OU complete response (ie, OU-free) and maintenance of OU complete response (ie, complete response at Week 6 and remaining OU-free for $\geq 6$ additional weeks); OU partial response (ie, OU reduction $\geq 50 \%$ ); disease activity (Behçet's Syndrome Activity Score [BSAS]; Behçet's Disease Current Activity Form [BDCAF], including Behçet's Disease Current Activity Index [BDCAI], and Patient's and Clinician's Perception of Disease Activity); and QoL (BDQoL; Short Form Health Survey version 2 [SF-36v2], including Physical Functioning [PF] scale and Physical and Mental Component Summary [PCS, MCS]).

Results: Of 207 patients randomized and treated in RELIEF, 52 were in the European subgroup. Mean $( \pm S D)$ age in the subgroup was $39( \pm 12)$ years; $54 \%$ were women. Baseline disease characteristics were similar between treatment groups (Table 1). Patients receiving APR achieved lower $A_{U C} C_{W k 0-12}$ for OU vs $\mathrm{PBO}$ (Figure 1) and greater reduction in pain. A greater proportion of patients receiving APR achieved complete, maintained, or partial OU responses at Week 12 vs those receiving PBO (Table 1). Consistent treatment effects favoring APR vs PBO were observed in disease activity, as shown by BSAS and BDCAF component scores at Week 12 (Table 1). Greater improvement in SF-36v2 MCS was observed favoring APR vs PBO at Week 12, and moderate treatment differences were seen for other QoL measures (BDQoL, SF-36v2 PF, and SF-36v2 PCS). Conclusion: In the European subgroup of patients with Behçet's syndrome and OU in RELIEF, APR resulted in greater reduction in OU count, OU pain, and disease activity as well as favorable treatment effect on QoL measures than PBO.
These results are consistent with the efficacy of APR treatment in the overall RELIEF population.

\begin{tabular}{|c|c|c|c|}
\hline Baseline Disease Characteristics, Mean* & $\begin{array}{c}\text { PBO } \\
(\mathrm{n}=27)\end{array}$ & $\begin{array}{c}\text { APR } \\
(n=25)\end{array}$ & \\
\hline Duration of $\mathrm{BD}$, years & 9.0 & 8.2 & \\
\hline OU count & 3.8 & 4.0 & \\
\hline OU pain (VAS 0-100) & 60.6 & 64.2 & \\
\hline BSAS $(0-100)$ & 38.7 & 41.4 & \\
\hline BDCAI (0-12) & 3.5 & 3.6 & \\
\hline BDQoL $(0-30)$ & 10.5 & 9.0 & \\
\hline Efficacy Outcomes at 12 Weeks ${ }^{*}$ & $\begin{array}{l}\text { PBO } \\
(\mathrm{n}=27)\end{array}$ & $\begin{array}{c}\text { APR } \\
(\mathrm{n}=25)\end{array}$ & $\begin{array}{c}\text { Treatment } \\
\text { Difference }[95 \% \mathrm{Cl}]\end{array}$ \\
\hline OU pain (VAS 0-100), mean $^{\dagger}$ & -17.7 & -48.7 & $-31.0[-44.7,-17.3]$ \\
\hline OU complete response, $\mathrm{n}(\%)^{\ddagger}$ & $4(14.8)$ & $16(64.0)$ & $51.5[29.8,73.3]$ \\
\hline OU maintained response, $\mathrm{n}(\%)^{\ddagger}$ & $1(3.7)$ & $8(32.0)$ & $26.7[7.4,46.0]$ \\
\hline OU partial response, $n(\%)^{\ddagger}$ & $11(40.7)$ & $21(84.0)$ & $46.0[23.9,68.0]$ \\
\hline BSAS $(0-100)^{+, \S}$ & -5.23 & -20.68 & $-15.5[-22.6,-8.3]$ \\
\hline BDCAI $(0-12)^{+, \S}$ & -0.0 & -1.4 & $-1.4[-2.2,-0.6]$ \\
\hline Patient's Perception of Disease Activity ${ }^{\dagger} \S$ & -0.4 & -1.6 & $-1.2[-2.1,-0.4]$ \\
\hline $\begin{array}{l}\text { Clinician's Overall Perception of Disease } \\
\text { Activity }{ }^{\dagger, \S}\end{array}$ & -0.6 & -1.7 & $-1.0[-1.7,-0.4]$ \\
\hline BDQoL $(0-30)^{\dagger, \S}$ & -1.25 & -2.37 & $-1.12[-3.8,1.5]$ \\
\hline SF-36v2 MCS $(0-100)^{\dagger, \S}$ & -2.1 & 4.2 & $6.3[2.2,10.4]$ \\
\hline
\end{tabular}

*ITT population. ${ }^{\dagger}$ LS mean of the change from baseline at Week $12 .{ }^{\ddagger}$ Non-responde imputation for missing data. ${ }^{\S}$ LOCF approach. All efficacy endpoints (except BDQoL) were significant at the level of $P<0.05$.

Acknowledgements : This study was funded by Celgene. Additional analyses were funded by Amgen Inc. Writing support was funded by Amgen Inc. and provided by Kristin Carlin, RPh, MBA, of Peloton Advantage, LLC, an OPEN Health company.

\section{Primary Endpoint: Area Under the Curve for the Number of OU From Baseline Through Week 12}

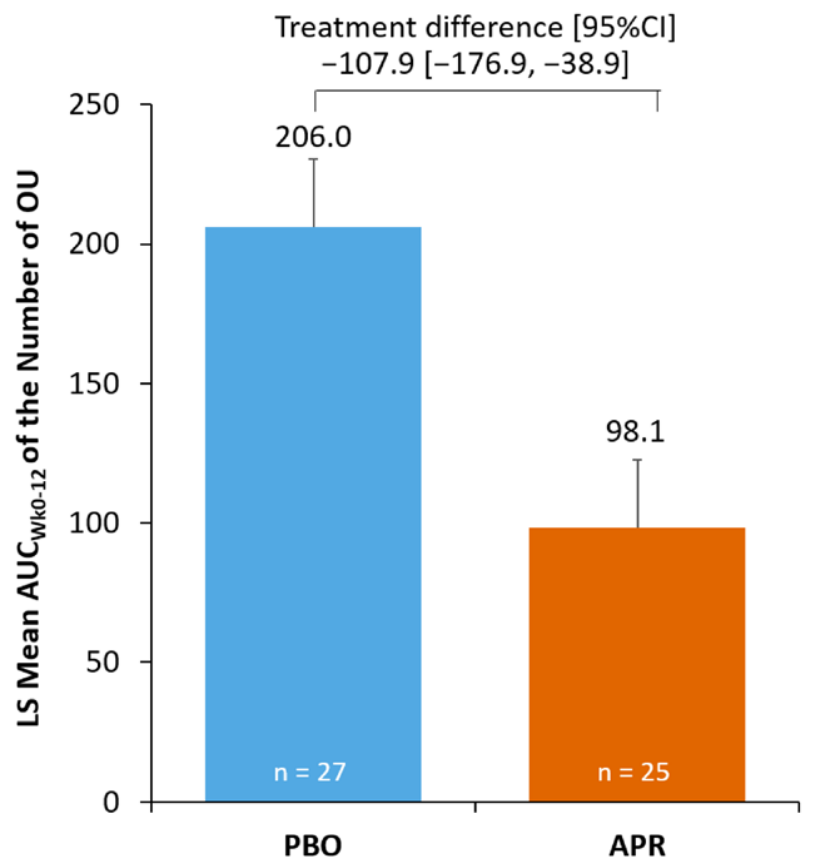

$P<0.05$; ITT population; error bars represent standard error. Multiple imputation used for imputing missing data.

Disclosure of Interests: Alfred Mahr Speakers bureau: Chugai; Roche, Consultant of: Celgene; Chugai, Gulen Hatemi Speakers bureau: AbbVie, Novartis, and UCB, Grant/research support from: Celgene, Mitsuhiro Takeno Speakers bureau: AbbVie, Esai, and Mitsubishi-Tanabe, Consultant of: Celgene, Grant/research support from: Novartis, Doyoung Kim: None declared, Melike Melikoglu: None declared, david Saadoun Consultant of: AbbVie, Celgene, Janssen, and Roche, Grant/research support from: AbbVie and Roche, Christos C. Zouboulis Speakers bureau: Amgen, Galderma, Pierre Fabre, PPM and Sobi, Consultant of: AbbVie AccureAcne, Almirall, Bayer Healthcare, GSK/Stiefel, Incyte, Inflarx, Janssen, Novartis, PPM, Regeneron, and UCB, Grant/research support from: Celgene NAOS-BIODERMA, and Relaxera, Sue Cheng Employee of: Amgen Inc, Sven Richter Employee of: Amgen Inc, Shauna Jardon Employee of: Amgen Inc, Maria 